\title{
Genome-Wide Screens Identify Group A Streptococcus Surface Proteins Promoting Female Genital Tract Colonization and Virulence
}

Luchang Zhu, ${ }^{* \dagger}$ Randall J. Olsen, ${ }^{* \dagger \dagger}$ Stephen B. Beres, ${ }^{* \dagger}$ Matthew Ojeda Saavedra, ${ }^{* \dagger}$ Samantha L. Kubiak, ${ }^{* \dagger}$ Concepcion C. Cantu, ${ }^{* \dagger}$ Leslie Jenkins, ${ }^{\S}$ Prasanti Yerramilli, ${ }^{\dagger}$ Layne Pruitt, ${ }^{* \dagger}$ Amelia R.L. Charbonneau, ${ }^{\varangle \| l}$ Andrew S. Waller, and James M. Musser ${ }^{\star \dagger \dagger}$

From the Center for Molecular and Translational Human Infectious Diseases Research* and the Department of Comparative Medicine, ${ }^{\S}$ Houston Methodist Research Institute, Houston, Texas; the Department of Pathology and Genomic Medicine, ${ }^{\dagger}$ Houston Methodist Hospital, Houston, Texas; the Department of Pathology and Laboratory Medicine, ${ }^{\ddagger}$ Weill Medical College of Cornell University, New York, New York; the Department of Bacteriology Research, " Animal Health Trust, Lanwades Park, Newmarket, United Kingdom; and the Department of Veterinary Medicine," University of Cambridge, Cambridge, United Kingdom

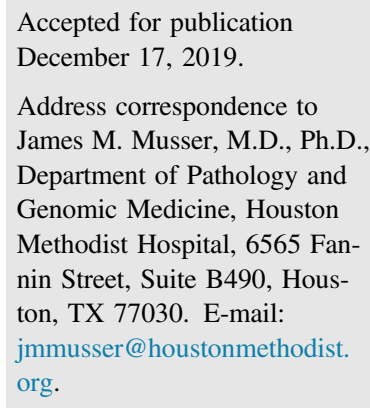

Group A streptococcus (GAS) is a major pathogen that impacts health and economic affairs worldwide. Although the oropharynx is the primary site of infection, GAS can colonize the female genital tract and cause severe diseases, such as puerperal sepsis, neonatal infections, and necrotizing myometritis. Our understanding of how GAS genes contribute to interaction with the primate female genital tract is limited by the lack of relevant animal models. Using two genome-wide transposon mutagenesis screens, we identified 69 GAS genes required for colonization of the primate vaginal mucosa in vivo and 96 genes required for infection of the uterine wall ex vivo. We discovered a common set of 39 genes important for GAS fitness in both environments. They include genes encoding transporters, surface proteins, transcriptional regulators, and metabolic pathways. Notably, the genes that encode the surface-exclusion protein (SpyAD) and the immunogenic secreted protein 2 (Isp2) were found to be crucial for GAS fitness in the female primate genital tract. Targeted gene deletion confirmed that isogenic mutant strains $\triangle$ spy $A D$ and $\triangle i s p 2$ are significantly impaired in ability to colonize the primate genital tract and cause uterine wall pathologic findings. Our studies identified novel GAS genes that contribute to female reproductive tract interaction that warrant translational research investigation. (Am J Pathol 2020, 190: 862-873; https://doi.org/10.1016/j.ajpath.2019.12.003)
Group A streptococcus (GAS or Streptococcus pyogenes) causes $>700$ million human infections worldwide and 15 million cases annually in the United States. ${ }^{1-3}$ Despite a century of research, no licensed vaccine is available. Although the oropharynx is the primary site of GAS infection, other mucosal surfaces can be colonized, sometimes leading to severe human infections and death., ${ }^{2,4,5}$ One example is GAS colonization of and proliferation in the female genital tract, a process that may result in puerperal sepsis (sometimes referred to as childbed fever) and/or neonatal infections. ${ }^{6-16}$ Although rare, puerperal sepsis and neonatal infections have high morbidity and mortality rates. ${ }^{9,11,14-16}$ The molecular mechanisms used by GAS to colonize the vaginal mucosal surface and to cause invasive infections in the uterine wall are poorly understood and thus somewhat mysterious and very murky. ${ }^{4,5}$ Given the rarity of these cases, studies designed to discover causal mechanisms in humans have been limited. However, recent work in nonhuman primates (NHPs) has found that new GAS

Supported in part by NIH grants AI 146771-01 (J.M.M.) and AI13936901 (J.M.M.); the Fondren Foundation of the Houston Methodist Hospital and Research Institute (J.M.M.); and University of Cambridge Doctoral Training Partnership scheme grant 1503883, funded by the Biotechnology and Biological Sciences Research Council (A.R.L.C.).

L.Z. and R.J.O. contributed equally to this work.

Disclosures: None declared. 
pathogenesis insight can be obtained using new relevant infection models. ${ }^{17}$

Transposon mutagenesis screens, such as conducted with TraDIS and Tnseq, are powerful tools to identify pathogen genes involved in the interaction with the host. ${ }^{18-29}$ Several genome-wide transposon mutagenesis screens have been successfully performed in GAS, resulting in the identification of novel genes important for growth in human blood and saliva ex vivo and for mouse invasive infections in vivo. ${ }^{23,30,31}$ We recently performed a systematic screen for GAS genes that contribute to necrotizing myositis in NHPs and identified many novel pathogen fitness genes with potential vaccine or therapeutic implications. ${ }^{17}$ Genome-wide study of the GAS genes that contribute to fitness in the female genital tract has not been undertaken but is warranted given the dearth of knowledge about pathogen-host interaction in this anatomic site.

One important major challenge for studying bacterial infections in the human female genital tract is the lack of a suitable experimental infection model. Recently, mouse models of vaginal colonization have been developed to study group B streptococcus and GAS genital infections. ${ }^{32-34}$ However, mouse infection models are unlikely to fully recapitulate primate genital tract interactions. In addition, an insufficient number of colony-forming units (CFUs) can be recovered from the mouse genital tract to generate statistically significant fitness gene data. In the current study, NHP models were developed for the study of upper and lower female genital tract interactions. These NHP female genital tract models are relevant experimental systems because GAS is a human-specific pathogen, many GAS virulence factors are specific for human and NHP targets, ${ }^{35-38}$ and the genital tract of NHPs is anatomically and microbiologically very similar to humans. ${ }^{39}$

GAS strains have been categorized historically into serotypes based on amino-terminal sequence and serologic variation in the $M$ protein, a surface-exposed virulence factor. ${ }^{40}$ Epidemiologic studies conducted over decades in many geographic locations have found that in contrast to the extensive variety of $\mathrm{M}$ protein serotypes that cause GAS pharyngitis and other infections, the spectrum of M serotypes that cause female genital tract infection is relatively narrow. Importantly, serotype M28 GAS strains are significantly overrepresented among cases of puerperal sepsis and neonatal GAS infections. ${ }^{9,10,41,42}$ The molecular mechanisms underpinning the enrichment of serotype M28 strains in genital infections are very poorly understood.

We report the first use of transposon mutagenesis screens to identify GAS genes that contribute to fitness in the female NHP genital tract. We developed two new NHP models of GAS pathogen-host interactions. Using a very dense transposon mutant library generated in a genetically representative serotype M28 GAS strain that commonly causes severe human infections, we discovered novel GAS genes required for colonization of the vaginal mucosa and contributing to disease pathogenesis of the uterine wall. It is reasonable to speculate that our findings have translational research implications.

\section{Materials and Methods}

\section{Bacterial Strains and Growth Conditions}

Serotype M28 GAS strain MGAS27961 is genetically representative of a clone that is prevalent in the United States and elsewhere. ${ }^{17,43}$ Strain MGAS27961 has wild-type (WT) alleles of all major transcriptional regulatory genes known to affect virulence, such as $\operatorname{cov} R, \operatorname{cov} S, \operatorname{rop} B, m g a$, and rocA. This strain was used to generate isogenic genedeletion mutant strains. GAS strains were grown in ToddHewitt broth supplemented with $0.5 \%$ yeast extract (THY).

\section{Preparation of Transposon Mutant Library Stock Cultures for NHP Infection}

A total of $100 \mu \mathrm{L}$ of the stock M28 GAS transposon mutant library was inoculated into $500 \mathrm{~mL}$ of THY supplemented with $0.5 \mu \mathrm{g} / \mathrm{mL}$ of erythromycin and cultured at $37^{\circ} \mathrm{C}$ for 8 hours. The expanded transposon mutant library was pelleted by centrifugation, washed three times with phosphatebuffered saline (PBS), and then suspended in $10 \mathrm{~mL}$ of PBS supplemented with $20 \%$ glycerol. The suspended mutant library was divided into 10 equivalent $1 \mathrm{~mL}$ aliquots, transferred to cryogenic tubes, and stored at $-80^{\circ} \mathrm{C}$ until NHP inoculation.

\section{NHP Vaginal Colonization Model}

A new NHP model of vaginal colonization was developed. For the transposon mutant library screens, six nulliparous, female cynomolgus macaques ( 2 to 3 years old and 2 to $4 \mathrm{~kg}$ ) were used. Before inoculation, NHPs received one dose of medroxyprogesterone acetate to halt ovarian function. Animals were sedated with ketamine, $10^{10} \mathrm{CFU}$ of the M28 input library was inoculated into the vaginal vault with a sterile transfer pipet, and the animal was maintained in the prone position for 5 minutes until the fluid was absorbed into the mucosa. Animals were sampled at 24 hours after infection. To analyze the output transposon mutant library, three vaginal swabs were collected by circumferentially sweeping the mucosa, transferred to $2 \mathrm{~mL}$ of THY supplemented with erythromycin, and plated onto Selective Streptococcus Agar (Becton, Dickinson and Company, Franklin Lakes, NJ) plates to collect the output library. Before incubation, $100 \mu \mathrm{L}$ was removed, serially diluted in sterile PBS, and plated to determine the number of CFUs in the output library.

\section{Ex Vivo Uterine Wall Infection Model}

To study GAS infection in the myometrium, a new ex vivo uterine wall infection model was developed. Nine NHP uteri were obtained at necropsy by dissecting the fallopian tubes, 
Table 1 Primers and Probes Used for Generating Isogenic Gene Deletion Mutants and for TaqMan Quantitative RT-PCR

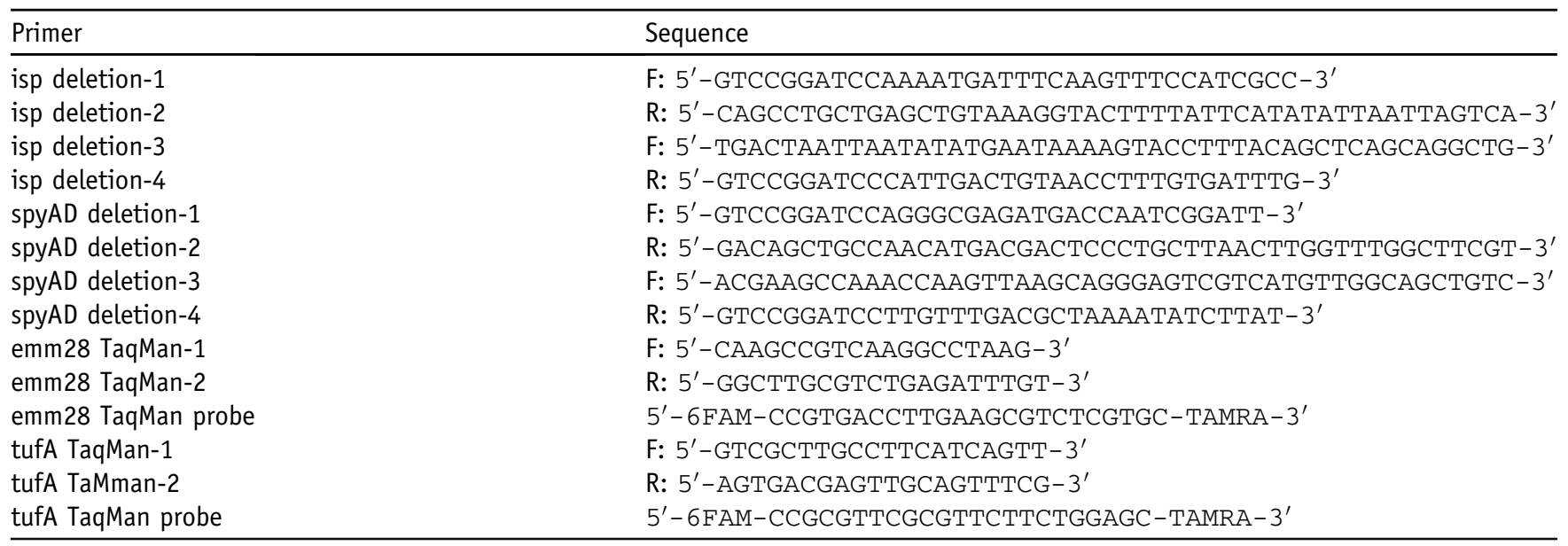

$F$, forward; R, reverse.

vagina, cervix, and attached soft tissues. Uteri were rinsed with sterile PBS and transferred to a sterile petri dish. The injection site for inoculation was marked, and $100 \mu \mathrm{L}$ of the transposon mutant library at a concentration of $3.5 \times 10^{10}$ $\mathrm{CFU} / \mathrm{mL}$ was injected into the myometrium. The infected uteri were transferred to $15-\mathrm{mL}$ tubes that contained $5 \mathrm{~mL}$ of Dulbecco's modified Eagle's medium (Thermo Fisher Scientific, Waltham, MA) supplemented with erythromycin and incubated at $37^{\circ} \mathrm{C}$ for 24 hours. Tissue was collected from the injection site using a sterile 8-mm biopsy punch, transferred to a $15-\mathrm{mL}$ tube that contained $1 \mathrm{~mL}$ of PBS, and homogenized (Omni International, Kennesaw, GA). Aliquots were plated on Selective Streptococcus Agar (Becton, Dickinson and Company) to count CFUs and prepare the output library for sequencing.

\section{Massively Parallel Sequencing and TraDIS Data Analysis}

The mutant pool genomic DNA preparation and transposon insertion site sequencing were performed according to previously described procedures. ${ }^{31}$ The resulting sequencing reads were analyzed with the TraDIS toolkit. ${ }^{44}$ The tradis_comparison.R script was used to compare the reads mapped per gene between the input pools and the output pools. ${ }^{44}$ The GAS genes with significantly decreased mutant frequency ( $\log 2$ foldchange $<-1$ and $q$ value $<0.1$ ) in the output transposon mutant pools were interpreted as required for GAS fitness. Illumina sequencing reads of the M28 GAS input and output libraries were deposited in the National Center for Biotechnology Information Sequence Read Archive (https://www. ncbi.nlm.nih.gov/sra; project number PRJNA596540).

\section{Construction and Characterization of Isogenic Deletion-Mutant Strains}

Isogenic deletion-mutant strains were derived from WT parental strain MGAS27961, the strain used for constructing the serotype M28 transposon insertion mutant library.
Primers used for generating the isogenic mutant strains are listed in Table 1. Isogenic mutant strains were constructed by nonpolar deletion of the target gene using allelic exchange. ${ }^{45}$ Whole genome sequencing of all isogenic mutant strains was performed to confirm the absence of spurious mutations.

\section{Quantitative RT-PCR Analysis}

Quantitative RT-PCR analysis of emm28 expression was performed according to previous descriptions. ${ }^{46,47}$ Briefly, GAS strains were grown in quadruplicate in THY broth to midexponential phase (OD600 $=0.5$ ). RNA was extracted from GAS culture using the RNeasy kit (Qiagen, Hilden, Germany). The purified RNA was converted to cDNA with Superscript III Reverse Transcriptase (Invitrogen, Carlsbad, CA). Quantitative PCR was performed using the TaqMan Fast Universal PCR Master Mix (Applied Biosystems, Foster City, CA). The sequences of the primers and probes used for quantitative PCR are listed in Table 1.

\section{Western Immunoblot Analysis of SPN and SLO}

Western immunoblot analysis of secreted toxins Streptococcus pyogenes $\mathrm{NAD}^{+}$-glycohydrolase (SPN) and streptolysin O (SLO) was performed as previously described. ${ }^{48}$ GAS strains were grown in THY broth to midexponential phase $(\mathrm{OD} 600=0.5)$. A total of $10 \mu \mathrm{L}$ of the culture supernatant from each strain was used for SDS-PAGE and subsequent immunoblotting.

\section{SpeB Protease Activity Assay}

SpeB protease activity was assessed according to previously described methods. ${ }^{49}$ GAS strains grown to midexponential phase were stabbed into milk agar plates, and the protease activity of each strain was analyzed after overnight incubation at $37^{\circ} \mathrm{C}$. 


\section{Vaginal Infection of NHPs with Isogenic Mutant} Strains

Following a similar protocol as used for the TraDIS screen, parental WT and isogenic mutant strains were compared in the NHP model of vaginal colonization. First, 12 nulliparous, female cynomolgus macaques ( 2 to 3 years old and 2 to $4 \mathrm{~kg}$ ) were used to compare the fitness of the WT and spyAD and isp2 isogenic deletion-mutant strains $(n=4$ animals per strain). All NHPs were given one dose of medroxyprogesterone acetate before inoculation. Animals were randomized to strain treatment groups, sedated with ketamine, inoculated in the vagina with $10^{9} \mathrm{CFU}$ of the indicated strain, and maintained in a prone position for 5 minutes until the fluid was absorbed into the mucosa. Animals were sampled on days $1,2,4$, and 7 after inoculation. At each time point, the vaginal mucosa was visually examined by a veterinarian using a bivalve nasal cone speculum, and three vaginal swabs were collected by circumferentially sweeping the vaginal mucosa. The swabs were transferred to $1 \mathrm{~mL}$ of PBS, serially diluted, and plated for counting.

\section{Infection of NHP Uteri ex Vivo with Isogenic Mutant Strains}

WT and isogenic mutant strains were compared using the ex vivo uterine wall infection model described above. Nine uteri extracted from female NHPs at necropsy were used to compare the WT and each of the two isogenic mutant strains ( $n=3$ uteri per strain). Each uterus was inoculated in the myometrium with $10^{9} \mathrm{CFU}$ of the indicated strain and incubated in Dulbecco's modified Eagle's medium for 24 hours. Tissue was collected from the injection site with a sterile 8-mm biopsy punch and homogenized to enumerate CFU counts. For histopathologic analysis, a full-thickness section of tissue taken from the inoculation site was fixed in $10 \%$ phosphate-buffered formalin, embedded in paraffin using standard automated instruments, and reviewed by a pathologist (R.J.O.) blinded to the strain treatment groups.

\section{Skeletal Muscle Infection of NHPs with Isogenic Mutant Strains}

The virulence of the parental WT strain and the two isogenic deletion-mutant strains was assessed in the NHP necrotizing myositis infection model. Animals were randomly assigned to different strain treatment groups and received $10^{8} \mathrm{CFU} / \mathrm{kg}$ of one strain (WT or isogenic mutant) in the lower limb. Each strain was tested in triplicate. The animals were observed continuously and necropsied at 24 hours after inoculation.

\section{Histopathologic Analysis}

For histologic evaluation, lesions were excised and visually inspected. Lesions (necrotic muscle) were measured in 3 dimensions, and volume was calculated using the formula
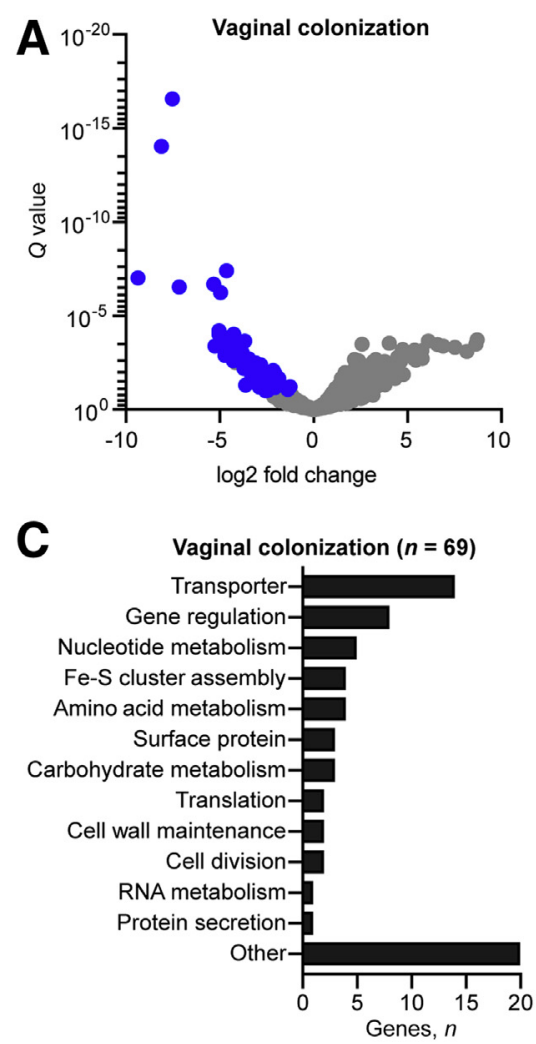
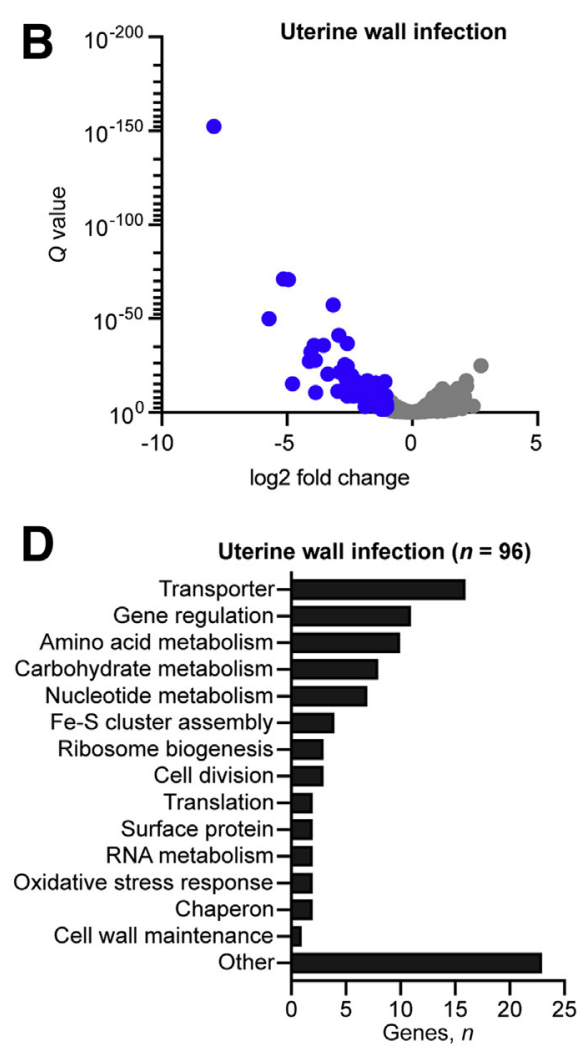

Figure 1 TraDIS analysis of group A streptococcus (GAS) gene fitness in female nonhuman primate (NHP) genital tract. A and B: Volcano-plot summary of the changes in mutant abundance for each of the genes in the output mutant pools. Gene mutations (transposon insertions) that confer significantly decreased fitness (blue circles) during vaginal colonization (A) and uterine smooth muscle wall infection (B) are highlighted. C and D: Functional categorization of the GAS fitness genes identified in vivo in the NHP female genital tract (C) and ex vivo in the uterine wall (D). 

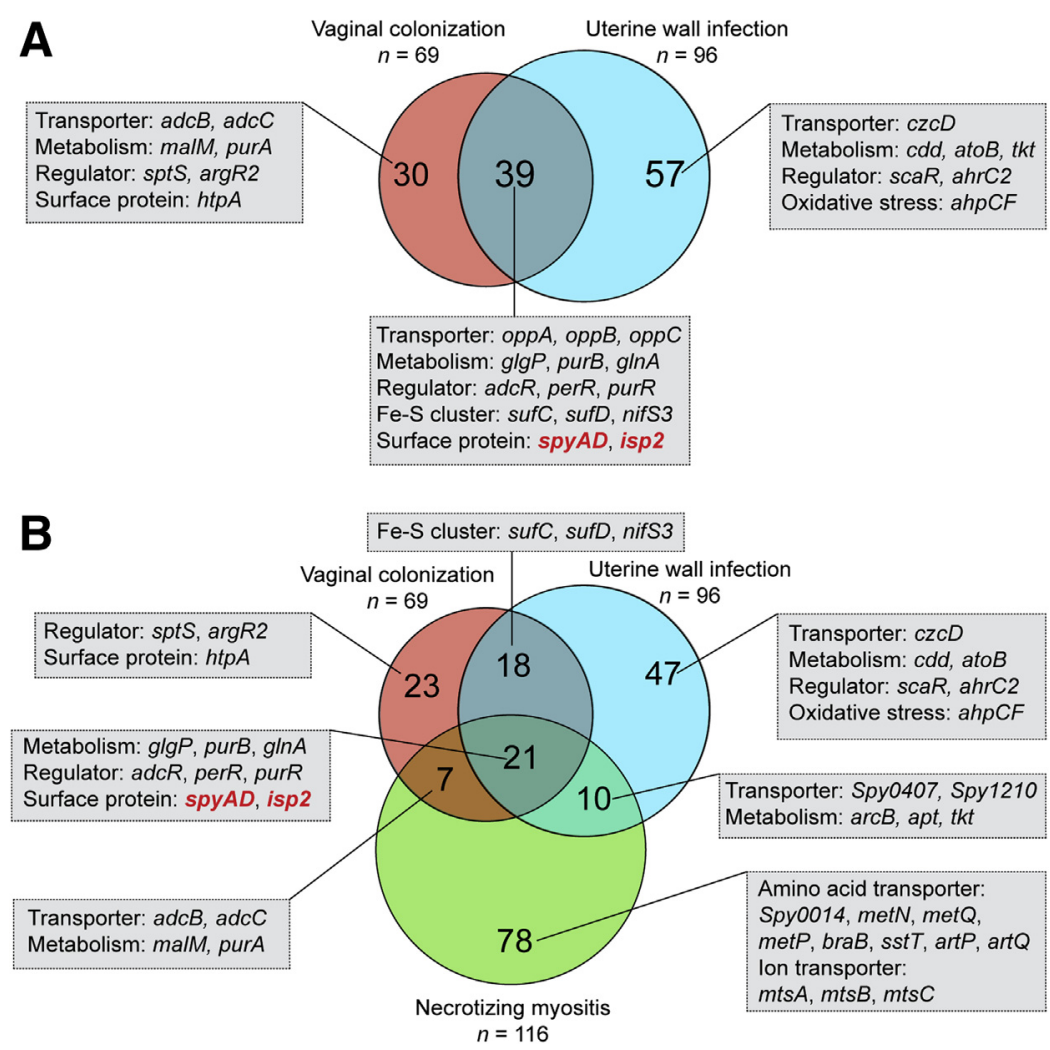

Figure 2 Group A streptococcus (GAS) genes that contribute to primate female genital tract mucosal infection and myometritis and skeletal muscle necrotizing myositis. A: Venn diagram comparison of 69 genes required for primate female genital tract mucosal infection with 96 genes required for uterine wall infection. B: Venn diagram comparison of GAS genes required for female genital tract infection with 116 genes required for necrotizing myositis. Representative fitness genes assigned to each category are shown. Genes selected for further validation and investigations are highlighted in red. for an ellipsoid. Tissue taken from the inoculation site was fixed in $10 \%$ phosphate-buffered formalin and embedded in paraffin using standard automated instruments as described previously. ${ }^{17}$ To obtain the quantitative CFU data, diseased muscle obtained from the inoculation site was weighed and homogenized (Omni International) in $1 \mathrm{~mL}$ of PBS. CFUs were determined by plating serial dilutions of the homogenate. Statistical differences between strain groups were determined using the $U$-test.

\section{Statistical Analysis}

Results of the CFU recovery from the NHP vaginal mucosa are expressed as means \pm SEM, with statistically significant differences determined using repeated-measures analysis of variance (Prism 6; GraphPad Software, San Diego, CA). Results of CFU recovery from the infected uteri or muscle are expressed as means \pm SEM, with statistically significant differences determined using the $U$-test (Prism 6). $P<0.05$ was considered statistically significant.

\section{Study Approvals}

All animal experiments were approved by the Institutional Animal Care and Use Committee of Houston Methodist Research Institute (protocol AUP-1217-0058).

\section{Results}

Genome-Wide Transposon Mutagenesis Screen Identifies GAS Genes Required for Vaginal Colonization and Uterine Wall Infection

We previously generated a highly saturated transposon insertion mutant library in MGAS27961, a serotype M28 strain that is genetically representative of a phylogenetic lineage that causes abundant human infections. ${ }^{43}$ To understand the molecular basis of GAS interaction with the vaginal mucosa, the M28 transposon mutant library in the female primate genital tract was screened to identify genes that contribute to GAS fitness in this host anatomic niche. Six female cynomolgus macaques were infected in the genital tract with the transposon mutant library. Swabs were obtained from the vagina of each animal 24 hours after inoculation. GAS cells recovered from the vaginal swabs constituted the output mutant pools. TraDIS was used to analyze the change in the composition of the output mutant pools relative to the input mutant pools. Transposon-inactivated genes associated with significantly decreased fitness (transposon frequency $\log 2$ fold change $<-1$ and $\mathrm{q}$ value $<0.1$ ) in the output mutant pools were interpreted as contributing to genital tract colonization. The TraDIS analysis identified 69 genes that contribute to GAS fitness in the vaginal mucosal surface of NHPs (Figures 1 and 2, Supplemental Table S1). 
Necrotizing myometritis is a devastating disease with very high morbidity and mortality rates. As a first step toward gaining information about GAS genes that contribute to this disease, an ex vivo uterine wall infection model was developed using explanted NHP uteri. Using this infection model, an analogous TraDIS screen was performed to identify GAS genes required for infection pathogenesis in the uterine wall ex vivo. Eight uteri recovered from NHPs at necropsy were infected in the uterine wall with the M28 GAS transposon mutant library. After 24 hours of incubation at $37^{\circ} \mathrm{C}$, GAS cells recovered from each infected uterus were collected as the output mutant pools. TraDIS analysis identified 96 genes that contribute to GAS fitness during uterine wall infection ex vivo (Figures 1 and 2 and Supplemental Table S2).

Functional categorization of the genes required for vaginal colonization and those required for uterine wall infection found that they encode various proven or putative transporters, transcriptional regulators, sensor kinase proteins, metabolic enzymes (nucleotide metabolism, amino acid metabolism, and carbohydrate metabolism), iron-sulfur cluster assembling proteins, and bacterial surface-displayed proteins (Figure 1).

\section{Comparison of GAS Genes Required for Vaginal Colonization, Uterine Wall Infection, and Skeletal Muscle Necrotizing Myositis}

Comparing the 69 vaginal tract fitness genes with the 96 uterine wall fitness genes found that a common set (ie, overlap) of 39 genes is required in both conditions (Figure 2A). This group includes genes that encode substrate transporters, metabolic enzymes, gene regulators, Fe-S cluster assembly proteins, and GAS secreted surfaceexposed protein (SpyAD) and immunogenic secreted protein 2 (Isp2). We recently identified 116 serotype M28 GAS genes required for bacterial fitness during NHP skeletal muscle necrotizing myositis. ${ }^{17}$ Comparing the fitness genes required for female genital tract infection (vaginal colonization and uterine wall infection) with the 116 necrotizing myositis genes found that most genital tract fitness genes did not overlap with genes required for necrotizing myositis (Figure 2B). Many genes were uniquely important in the female genital tract environments, including encoding sensor kinase SptS, transcriptional regulator ArgR2, and iron-sulfur cluster assembly proteins (Figure 2B). Conversely, 21 genes are required for both genital tract infection and necrotizing myositis, including genes encoding metabolic enzymes $(g \lg P$, purB, and $g \ln A)$, transcriptional regulators (adcR, perR, and purR), and bacterial surface-exposed proteins (spyAD and isp2) (Figure 2B). Seventy-eight GAS genes required for necrotizing myositis were found to be likely dispensable for female genital tract colonization, including genes for a variety of amino acid transporters (Spy0014, metQNP, braB, and sstT) and a manganese transporter ( $m t s A B C$ ) (Figure 2B).
Contribution of SpyAD and Isp2 to GAS Fitness in the NHP Genital Tract Environments

Bacterial surface-exposed proteins are attractive therapeutic and vaccine targets. Our TraDIS screen results suggest genes encoding SpyAD and Isp2, two GAS surface proteins, ${ }^{50-52}$ contribute to the GAS fitness in the female genital tract. To definitively determine whether SpyAD and Isp2 participate in GAS colonization of the primate female genital tract, isogenic gene deletion mutant strains $\triangle$ spyAD and $\Delta i s p 2$ were generated in parental strain MGAS27961 (Figure 3A). The two isogenic mutants do not have a growth defect in rich medium THY (Figure 3B). Compared with the WT parental strain, the spyAD and isp2 deletion mutants do not have a statistically significant change in the transcript level of emm28, the gene encoding antiphagocytic M pro$\operatorname{tein}^{53}$ (Figure 3C). In addition, the two isogenic mutants do not have a marked change in the production of secreted virulence factors SPN, SLO, and cysteine protease SpeB (Figure 3, D and E). Four female NHPs were infected each in the genital tract with parental strain MGAS27961, the isogenic spyAD or isp2 deletion-mutant strain. Compared with the parental strain, both isogenic mutant strains were significantly impaired in capacity to colonize the NHP vaginal mucosa (Figure $4 \mathrm{~A}$ ).

To validate the role of the spyAD and isp2 genes to GAS fitness in the uterine wall infection model, the growth of each of the spyAD and isp2 isogenic mutant strains in the myometrium of explanted NHP uteri was examined. Consistent with the TraDIS screen results, the isogenic mutants $\triangle$ spyAD and $\Delta i s p 2$ were significantly attenuated for growth in the uterine wall (Figure 4B). The WT strain caused large foci of bacterial growth associated with necrotic myometrium, whereas the isogenic mutants $\triangle$ spyAD and $\Delta i s p 2$ caused a markedly smaller area of myometrium destruction (Figure $4 \mathrm{C}$ ). Taken together, these data confirm that $s p y A D$ and $i s p 2$ contribute significantly to GAS fitness in these two NHP genital tract environments.

\section{SpyAD and Isp2 Contribute Significantly to GAS Necrotizing Myositis}

In addition to their contribution to genital tract infections, the results of our previous genome-wide screens suggest that spyAD and isp2 also contribute to GAS fitness during necrotizing myositis. ${ }^{17}$ To determine whether SpyAD and Isp2 also play a role in necrotizing myositis, the virulence of the parental WT strain and isogenic gene-deletion mutant strains was assessed using a NHP necrotizing myositis infection model. ${ }^{54}$ Consistent with the initial genome-wide screen result, ${ }^{17}$ isogenic mutant strains $\triangle$ spyAD and $\Delta i s p 2$ were significantly attenuated in causing necrotizing myositis in NHPs. Compared with the WT parental strain, significantly fewer CFUs of each isogenic mutant strain were recovered from the inoculation site (Figure 5A). In addition, compared with the WT parental strain, each of the two 
A

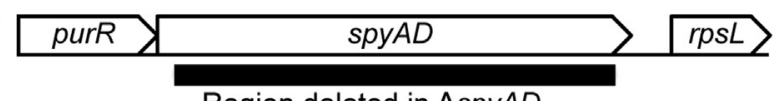

Region deleted in $\triangle$ spyAD
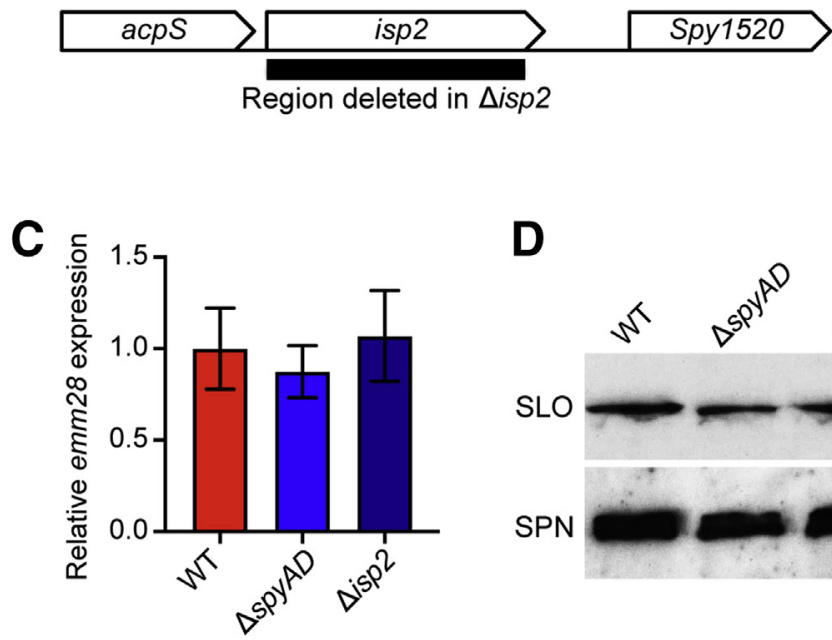

D

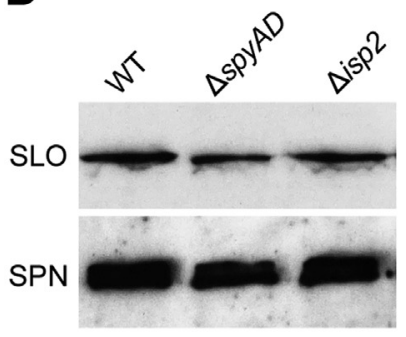

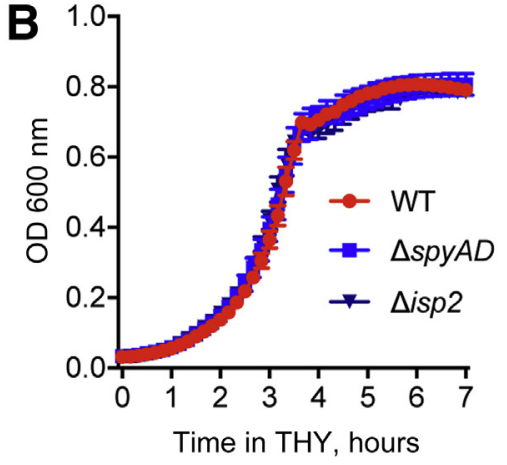

$\mathbf{E}$

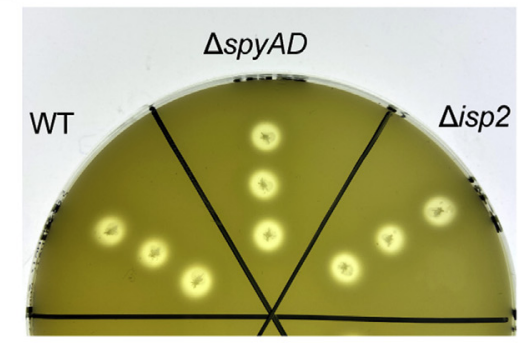

Figure 3 In vitro phenotypes of the isogenic gene deletion mutants. A: Schematic showing the gene neighborhood of spyAD and isp2 genes and the deleted regions of isogenic mutants $\triangle$ spy $A D$ and $\triangle i s p 2$. B: The isogenic gene-deletion mutant strains have no significant growth defect in rich medium ToddHewitt broth supplemented with $0.5 \%$ yeast extract (THY). C: Isogenic mutant strains $\Delta$ spyAD and $\Delta i s p 2$ have no significant change in emm28 transcript level. D and E: The two isogenic mutant strains do not have a marked change in the production of secreted toxins Streptococcus pyogenes NAD ${ }^{+}$-glycohydrolase $(\mathrm{SPN})$, streptolysin 0 (SLO) (shown by Western immunoblotting), and cysteine protease SpeB (shown by SpeB protease activity assay). $n=4$ (one-way analysis of variance). WT, wild type.

mutant strains caused significantly smaller lesions characterized by less tissue destruction (Figure 5, B and C).

\section{Genes Encoding SpyAD and Isp2 Are Ubiquitous in GAS Isolates}

The distribution of the spyAD and isp2 genes was examined in i) 200 closed GAS genomes that represented $77 \mathrm{emm}$ types in the National Center for Biotechnology Information microbial genome database (www.ncbi.nlm.nih.gov/genomel microbes) as of August 1, 2019, ii) the CDC's Active Bacterial Core Surveillance invasive GAS isolates that covered $61 \mathrm{emm}$ types from $2015,{ }^{55}$ and iii) our intercontinental collection of 2,095 serotype M28 GAS strains. ${ }^{43}$ Using the Basic Local Alignment Search Tool (BLAST), it was found that $s p y A D$ and $i s p 2$ are present in $100 \%$ of the GAS strains of the three populations mentioned above (Supplemental Table S3).

\section{Discussion}

Little is known about GAS molecular pathogenesis in the female lower and upper genital tract. Mouse models of infection have been used relatively recently to study GAS-female genital tract interactions. For example, mouse infection models found that IL-17A contributes to the control of GAS vaginal colonization. ${ }^{56}$ Leukotriene B4 plays an important role in GAS puerperal sepsis. ${ }^{57}$ A transcriptome study using a mouse model identified a GAS surface protein that contributed to vaginal colonization. ${ }^{58}$ However, because of phylogenetic distance and anatomic and immunologic differences, it is unclear whether mouse models of infection adequately recapitulate GAS infections in humans. To address this knowledge deficit, we developed two new NHP infection models and performed genome-wide screens that successfully identified GAS genes that contribute to colonization of the NHP vaginal tract and infection in the uterine wall.

A significant portion of the identified GAS fitness genes that contribute to female NHP genital tract colonization and uterine wall infection encode proven or putative transporters (Figure 1), including transporters for metal ions ( $a d c B$, $a d c C$ ), and oligopeptides (oppABCDF) (Figure 2). We recently found that GAS transporters also play a pivotal role during experimental NHP skeletal muscle necrotizing myositis. ${ }^{17}$ Collectively, these results suggest that nutrient acquisition from the host is fundamental for GAS to colonize and establish infections in these anatomic sites. GAS transporters that contribute to colonization and infection warrant further study for potential vaccine or therapeutic use.

GAS can colonize and infect multiple distinct human anatomic sites. It is reasonable to speculate that the spectrum of genes required for GAS to grow on the vaginal 

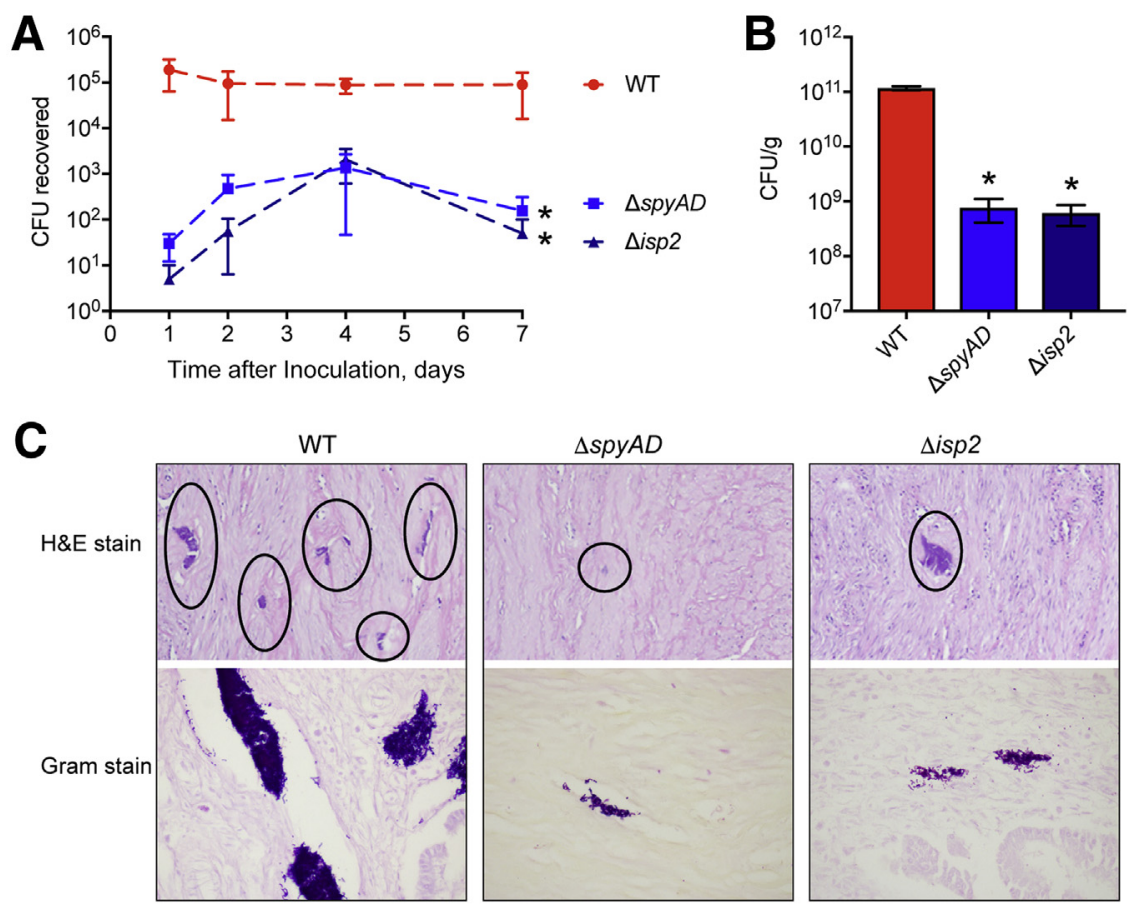

Figure 4 Validation of the contribution of spyAD and isp2 to group A streptococcus (GAS) fitness in the nonhuman primate (NHP) genital tract. A: The ability of wild-type (WT) parental strain and isogenic mutant strains $\triangle$ spyAD and $\Delta i s p 2$ to colonize NHP vaginal epithelial mucosa in vivo. B: Growth of the WT parental and isogenic mutant strains in the NHP uterine wall ex vivo. C: Micrographs of uterine wall lesions caused by the parental WT strain and isogenic gene-deletion mutant strains. The WT strain is associated with larger foci of necrotic myometrium with higher numbers of organisms in the NHP uterine wall ex vivo (circled areas). Data are expressed as means \pm SEM (A and B). $n=4(\mathbf{A}) ; n=3$ (B). ${ }^{*} P<0.05$ versus WT (Kruskal-Wallis test or $U$ test). Original magnification: $\times 4$ (C, top row); $\times 40$ (C, bottom row). H\&E, hematoxylin and eosin. mucosa, in the uterine wall, and in the skeletal muscle might be different. To identify potential niche-specific genes critical for GAS fitness in the female genital tract environments, the GAS genes required for NHP vaginal tract colonization and uterine wall infection were first compared (Figure 2A).
The results indicate that $39(57 \%)$ of the same genes required for vaginal mucosal colonization are also important for uterine wall infection, suggesting some similarity between the vaginal mucosa and myometrium environments. Conversely, the comparison also identified genes that are
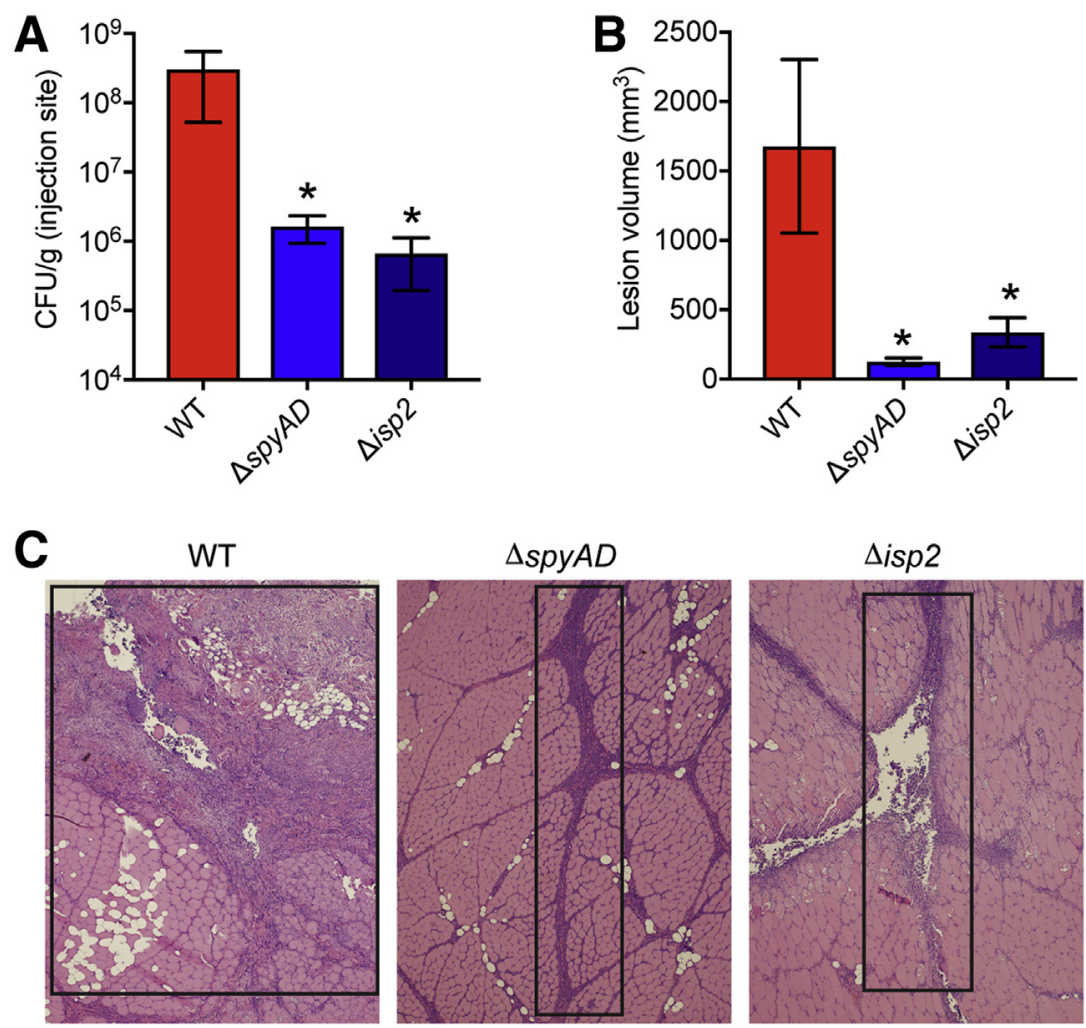

Figure 5 Validation of the contribution of spyAD and isp2 to group A streptococcus (GAS) virulence during necrotizing myositis. A: Colony-forming units (CFUs) recovered from the necrotizing myositis lesions caused by wild-type (WT) parental and isogenic mutant strains in nonhuman primate (NHP). B: Volume of necrotizing myositis lesions caused by WT and each isogenic gene-deletion mutant strain. C: Micrographs of necrotizing myositis lesions caused by the parental WT strain and isogenic gene-deletion mutant strains (hematoxylin and eosin staining). The WT strain causes larger lesions with more tissue destruction (boxed areas). Data are expressed as means $\pm \operatorname{SEM}(\mathbf{A}$ and $\mathbf{B})$. $n=4$ (A and $\mathbf{B}) .{ }^{*} P<0.05$ versus WT ( $U$-test). Original magnification, $\times 4$. 
uniquely important for vaginal colonization or for causing uterine wall pathologic findings. For example, genes for zinc acquisition $(a d c B, a d c C$, and $h t p A)$ are important for vaginal colonization, whereas the gene that encode zinc efflux $(c z c D)$ is required for GAS fitness in the myometrium, suggesting that zinc availability differs between the vaginal mucosa and myometrium. Interestingly, a recent study using mouse models also found that GAS zinc efflux and zinc import are important in different anatomic niches. ${ }^{59}$ GAS genes required for the two genital tract environments were next compared with those that contribute to NHP skeletal muscle necrotizing myositis (Figure 2B). The results indicate that the two-component regulatory system sensor kinase $\mathrm{SptS},{ }^{60}$ transcriptional regulator ArgR2, and $\mathrm{Fe}-\mathrm{S}$ cluster assembly proteins are required for GAS genital tract infections but not for skeletal muscle fitness (Figure 2B). Conversely, amino acid transporters (Spy0014, $m e t Q N P$, braB, and $s s t T$ ) are critical for GAS survival during skeletal muscle necrotizing myositis but less so for GAS genital tract infections (Figure 2B). Further studies using isogenic gene deletion mutants are necessary to validate these observations. The comparison also identified a set of 21 GAS genes that are required in each of the three infection models (Figure 2B). This set of genes warrants further study as translational research targets.

It was discovered that two GAS surface-displayed proteins, Isp2 and SpyAD, contribute significantly to GAS colonization of the NHP female genital tract. The two genes are members of the 21 genes that contribute to fitness in the three NHP infection models. Use of isogenic mutant strains documented that Isp2 and SpyAD are critical for GAS to cause necrotizing myositis in NHPs (Figure 4). ${ }^{17}$ Proteomic analysis found that Isp2 is one of the most abundant secreted extracellular proteins made by GAS. ${ }^{61}$ Isp2 has a CHAP domain, ${ }^{62}$ which is often present in enzymes involved in peptidoglycan hydrolysis, suggesting that Isp2 plays a role in bacterial cell wall modification, but this has not been proven. SpyAD is a GAS cell wall protein that has been investigated as a vaccine candidate. ${ }^{63,64}$ Vaccination of mice with purified recombinant SpyAD confers significant protection against intranasal infection with multiple GAS serotypes. ${ }^{63,65}$ SpyAD has been proposed to function as an adhesin. ${ }^{51}$ However, the precise mechanism of SpyAD and Isp2 interaction with the female genital tract is unclear but is under investigation. Analysis of publicly available GAS genomes, the CDC's collection of invasive GAS strains, and our own collection of strains found that genes that encode SpyAD and Isp2 are ubiquitously present in GAS isolates (Supplemental Table S3). Consistent with our observation, a previous study found that the spyAD gene is present in $>99 \%$ of isolates in a large global GAS collection. ${ }^{66}$ The high gene presence rate, surface localization, and roles in virulence make SpyAD and Isp2 attractive vaccine and therapeutic targets.

Serotype M28 GAS strains have a 37.4-Kb integrativeconjugative element designated as region of difference 2 (RD2). ${ }^{41,67}$ Close homologs of several surface proteins that encode genes in RD2 (such as the GAS surface protein and the R28 protein) are also present in many group B streptococcus strains, ${ }^{68,69}$ a major cause of female genital tract and neonatal infections. A recent study reported that deletion of the entire RD2 region conferred decreased ability of an M28 GAS strain to adhere in vitro to immortalized human vaginal epithelial cells and to colonize the female genital tract in a mouse model of infection. ${ }^{70}$ Our screen did not identify genes in the RD2 region as contributing factors for GAS colonization in this NHP female genital tract infection model. At least three possibilities may explain this observation. First, only genes that contribute to GAS fitness during the first 24 hours of infection in the NHP vagina were screened. If genes in the RD2 region contribute to the long-term persistence of GAS in this site, this screen may not detect them. Second, the nature of our transposon mutagenesis screens is mixed infections. If a determinant that contributes to GAS fitness is a secreted protein, our screen may not identify this factor because of transcomplementation of mutant strains by products made by other strains in the inoculum that retains a WT phenotype for genes in the RD2 element. This finding may be an especially important matter in the context of RD2, an element that encodes seven secreted proteins. ${ }^{41,69} \mathrm{~A}$ third possibility is that cell culture and mouse models simply do not adequately recapitulate the female primate condition. Future studies using the NHP vaginal model of infection and isogenic gene deletion mutants are needed to definitively clarify the contribution of RD2 element genes to M28 GAS female genital tract infections.

To summarize, we developed two new NHP models for the study of GAS infection biology and used transposon mutant library screens to identify pathogen genes that contribute to colonization of the NHP vaginal tract mucosa and infection in the uterine wall. Using isogenic mutant strains, it was confirmed that genes encoding two bacterial surface proteins (Isp2 and SpyAD) are crucial for GAS vaginal colonization, myometrium infection, and necrotizing myositis. Our findings may lead to better ways to treat female GAS genital tract infections. We believe the NHP models of genital tract infection and transposon mutagenesis screens could be used to discover fitness and virulence determinants of other important female genital tract pathogens, such as group B streptococcus. Together, such studies could also make important contributions to our understanding of the molecular basis of puerperal sepsis, a disease that has an unusually rich history in medicine because of Semmelweis' pioneering studies. ${ }^{71,72}$

\section{Acknowledgments}

We thank Drs. Kjersti M. Aagaard, Frank R. DeLeo, and Dominique Missiakas for critical reading of the manuscript and suggesting improvements; animal husbandry personnel for outstanding veterinary technical assistance; and Dr. Sasha M. Pejerrey and Adrienne Winston for editorial assistance. 


\section{Author Contributions}

L.Z. designed the studies, performed and analyzed TraDIS experiments, constructed and characterized isogenic mutant strains, and wrote the manuscript; R.J.O. planned and conducted experiments involving the NHPs, analyzed resulting data, and wrote the manuscript; M.O.S. sequenced the isogenic mutant strains and provided technical support for the NHP studies; S.L.K., P.Y., and L.P. constructed and characterized isogenic mutant strains; C.C.C. provided extensive technical support for all phases of the study; A.R.L.C. and A.S.W. provided intellectual guidance on the TraDIS data analysis; L.J. oversaw and performed the NHP experiments; J.M.M. designed the studies, analyzed experiments, wrote the manuscript, and oversaw the project; L.Z. and R.J.O. made substantial scientific contributions to this work and share the first author byline position.

\section{Supplemental Data}

Supplemental material for this article can be found at http://doi.org/10.1016/j.ajpath.2019.12.003.

\section{References}

1. Bisno AL: Acute pharyngitis. N Engl J Med 2001, 344:205-211

2. Carapetis JR, Steer AC, Mulholland EK, Weber M: The global burden of group A streptococcal diseases. Lancet Infect Dis 2005, 5: 685-694

3. Wessels MR: Clinical practice: streptococcal pharyngitis. N Engl J Med 2011, 364:648-655

4. Walker MJ, Barnett TC, McArthur JD, Cole JN, Gillen CM, Henningham A, Sriprakash KS, Sanderson-Smith ML, Nizet V: Disease manifestations and pathogenic mechanisms of group A streptococcus. Clin Microbiol Rev 2014, 27:264-301

5. Wilkening RV, Federle MJ: Evolutionary constraints shaping Streptococcus pyogenes-host interactions. Trends Microbiol 2017, 25: $562-572$

6. Colebrook L: Infection by anaerobic streptococci in puerperal fever. Br Med J 1930, 2:134-137

7. Hughes BL: Group A streptococcus puerperal sepsis: an emerging obstetric infection? BJOG 2019, 126:54

8. Lancefield RC, Hare R: The serological differentiation of pathogenic and non-pathogenic strains of hemolytic streptococci from parturient women. J Exp Med 1935, 61:335-349

9. Chuang I, Van Beneden C, Beall B, Schuchat A: Population-based surveillance for postpartum invasive group a streptococcus infections, 1995-2000. Clin Infect Dis 2002, 35:665-670

10. Colman G, Tanna A, Efstratiou A, Gaworzewska ET: The serotypes of streptococcus pyogenes present in Britain during 1980-1990 and their association with disease. J Med Microbiol 1993, 39:165-178

11. Eriksson BK, Norgren M, McGregor K, Spratt BG, Normark BH: Group A streptococcal infections in Sweden: a comparative study of invasive and noninvasive infections and analysis of dominant T28 emm28 isolates. Clin Infect Dis 2003, 37:1189-1193

12. Gaworzewska E, Colman G: Changes in the pattern of infection caused by Streptococcus pyogenes. Epidemiol Infect 1988, 100: 257-269

13. Lewis RF: Beta-haemolytic streptococci from the female genital tract: clinical correlates and outcome of treatment. Epidemiol Infect 1989, 102:391-400
14. O'Brien KL, Beall B, Barrett NL, Cieslak PR, Reingold A, Farley MM, Danila R, Zell ER, Facklam R, Schwartz B, Schuchat A: Epidemiology of invasive group a streptococcus disease in the United States, 1995-1999. Clin Infect Dis 2002, 35:268-276

15. Tyrrell GJ, Lovgren M, Forwick B, Hoe NP, Musser JM, Talbot JA: M types of group A streptococcal isolates submitted to the National Centre for Streptococcus (Canada) from 1993 to 1999. J Clin Microbiol 2002, 40:4466-4471

16. Vlaminckx B, van Pelt W, Schouls L, van Silfhout A, Elzenaar C, Mascini E, Verhoef J, Schellekens J: Epidemiological features of invasive and noninvasive group A streptococcal disease in the Netherlands, 1992-1996. Eur J Clin Microbiol Infect Dis 2004, 23 : 434-444

17. Zhu L, Olsen RJ, Beres SB, Eraso JM, Saavedra MO, Kubiak SL, Cantu CC, Jenkins L, Charbonneau ARL, Waller AS, Musser JM: Gene fitness landscape of group A streptococcus during necrotizing myositis. J Clin Invest 2019, 129:887-901

18. Armbruster CE, Forsyth-DeOrnellas V, Johnson AO, Smith SN, Zhao L, Wu W, Mobley HLT: Genome-wide transposon mutagenesis of Proteus mirabilis: essential genes, fitness factors for catheter-associated urinary tract infection, and the impact of polymicrobial infection on fitness requirements. PLoS Pathog 2017, 13:e1006434

19. Gawronski JD, Wong SM, Giannoukos G, Ward DV, Akerley BJ: Tracking insertion mutants within libraries by deep sequencing and a genome-wide screen for Haemophilus genes required in the lung. Proc Natl Acad Sci U S A 2009, 106:16422-16427

20. Subashchandrabose S, Smith SN, Spurbeck RR, Kole MM, Mobley HL: Genome-wide detection of fitness genes in uropathogenic Escherichia coli during systemic infection. PLoS Pathog 2013, 9:e1003788

21. Wang N, Ozer EA, Mandel MJ, Hauser AR: Genome-wide identification of Acinetobacter baumannii genes necessary for persistence in the lung. mBio 2014, 5. e01163-14

22. Weerdenburg EM, Abdallah AM, Rangkuti F, Abd El Ghany M, Otto TD, Adroub SA, Molenaar D, Ummels R, Ter Veen K, van Stempvoort G, van der Sar AM, Ali S, Langridge GC, Thomson NR, Pain A, Bitter W: Genome-wide transposon mutagenesis indicates that Mycobacterium marinum customizes its virulence mechanisms for survival and replication in different hosts. Infect Immun 2015, 83: $1778-1788$

23. Le Breton Y, Mistry P, Valdes KM, Quigley J, Kumar N, Tettelin H, McIver KS: Genome-wide identification of genes required for fitness of group A streptococcus in human blood. Infect Immun 2013, 81: $862-875$

24. Zhang X, de Maat V, Guzman Prieto AM, Prajsnar TK, Bayjanov JR, de Been M, Rogers MRC, Bonten MJM, Mesnage S, Willems RJL, van Schaik W: RNA-seq and Tn-seq reveal fitness determinants of vancomycin-resistant Enterococcus faecium during growth in human serum. BMC Genomics 2017, 18:893

25. Shames SR, Liu L, Havey JC, Schofield WB, Goodman AL, Roy CR: Multiple Legionella pneumophila effector virulence phenotypes revealed through high-throughput analysis of targeted mutant libraries. Proc Natl Acad Sci U S A 2017, 114:E10446-E10454

26. Gutierrez MG, Yoder-Himes DR, Warawa JM: Comprehensive identification of virulence factors required for respiratory melioidosis using Tn-seq mutagenesis. Front Cell Infect Microbiol 2015, 5:78

27. Palace SG, Proulx MK, Lu S, Baker RE, Goguen JD: Genome-wide mutant fitness profiling identifies nutritional requirements for optimal growth of Yersinia pestis in deep tissue. mBio 2014, 5:e1385-14

28. McCarthy AJ, Stabler RA, Taylor PW: Genome-wide identification by transposon insertion sequencing of Escherichia coli K1 genes essential for in vitro growth, gastrointestinal colonizing capacity, and survival in serum. J Bacteriol 2018, 200:e00698-17

29. Wilde AD, Snyder DJ, Putnam NE, Valentino MD, Hammer ND, Lonergan ZR, Hinger SA, Aysanoa EE, Blanchard C, Dunman PM, Wasserman GA, Chen J, Shopsin B, Gilmore MS, Skaar EP, 
Cassat JE: Bacterial hypoxic responses revealed as critical determinants of the host-pathogen outcome by TnSeq analysis of Staphylococcus aureus invasive infection. PLoS Pathog 2015, 11: e1005341

30. Le Breton Y, Belew AT, Freiberg JA, Sundar GS, Islam E, Lieberman J, Shirtliff ME, Tettelin H, El-Sayed NM, McIver KS: Genome-wide discovery of novel M1T1 group A streptococcal determinants important for fitness and virulence during soft-tissue infection. PLoS Pathog 2017, 13:e1006584

31. Zhu L, Charbonneau ARL, Waller AS, Olsen RJ, Beres SB, Musser JM: Novel genes required for the fitness of Streptococcus pyogenes in human saliva. mSphere 2017, 2:e00147-00117

32. Patras KA, Doran KS: A murine model of group B streptococcus vaginal colonization. J Vis Exp 2016, 117:1-26

33. Andrade EB, Magalhaes A, Puga A, Costa M, Bravo J, Portugal CC, Ribeiro A, Correia-Neves M, Faustino A, Firon A, Trieu-Cuot P, Summavielle T, Ferreira P: A mouse model reproducing the pathophysiology of neonatal group B streptococcal infection. Nat Commun 2018, 9:3138

34. Watson ME Jr, Nielsen HV, Hultgren SJ, Caparon MG: Murine vaginal colonization model for investigating asymptomatic mucosal carriage of Streptococcus pyogenes. Infect Immun 2013, 81: $1606-1617$

35. Sun H, Ringdahl U, Homeister JW, Fay WP, Engleberg NC, Yang AY, Rozek LS, Wang X, Sjobring U, Ginsburg D: Plasminogen is a critical host pathogenicity factor for group A streptococcal infection. Science 2004, 305:1283-1286

36. Sun H, Wang X, Degen JL, Ginsburg D: Reduced thrombin generation increases host susceptibility to group A streptococcal infection. Blood 2009, 113:1358-1364

37. Kasper KJ, Zeppa JJ, Wakabayashi AT, Xu SX, Mazzuca DM, Welch I, Baroja ML, Kotb M, Cairns E, Cleary PP, Haeryfar SM, McCormick JK: Bacterial superantigens promote acute nasopharyngeal infection by Streptococcus pyogenes in a human MHC class IIdependent manner. PLoS Pathog 2014, 10:e1004155

38. Sriskandan S, Unnikrishnan M, Krausz T, Dewchand H, Van Noorden S, Cohen J, Altmann DM: Enhanced susceptibility to superantigen-associated streptococcal sepsis in human leukocyte antigen-DQ transgenic mice. J Infect Dis 2001, 184:166-173

39. Ma J, Prince AL, Bader D, Hu M, Ganu R, Baquero K, Blundell P, Alan Harris R, Frias AE, Grove KL, Aagaard KM: High-fat maternal diet during pregnancy persistently alters the offspring microbiome in a primate model. Nat Commun 2014, 5:3889

40. Cunningham MW: Pathogenesis of group A streptococcal infections. Clin Microbiol Rev 2000, 13:470-511

41. Green NM, Zhang S, Porcella SF, Nagiec MJ, Barbian KD, Beres SB, LeFebvre RB, Musser JM: Genome sequence of a serotype M28 strain of group a streptococcus: potential new insights into puerperal sepsis and bacterial disease specificity. J Infect Dis 2005, 192: 760-770

42. Green NM, Beres SB, Graviss EA, Allison JE, McGeer AJ, VuopioVarkila J, LeFebvre RB, Musser JM: Genetic diversity among type emm28 group A Streptococcus strains causing invasive infections and pharyngitis. J Clin Microbiol 2005, 43:4083-4091

43. Kachroo P, Eraso JM, Beres SB, Olsen RJ, Zhu L, Nasser W, Bernard PE, Cantu CC, Saavedra MO, Arredondo MJ, Strope B, Do H, Kumaraswami M, Vuopio J, Grondahl-Yli-Hannuksela K, Kristinsson KG, Gottfredsson M, Pesonen M, Pensar J, Davenport ER, Clark AG, Corander J, Caugant DA, Gaini S, Magnussen MD, Kubiak SL, Nguyen HAT, Long SW, Porter AR, DeLeo FR, Musser JM: Integrated analysis of population genomics, transcriptomics and virulence provides novel insights into Streptococcus pyogenes pathogenesis. Nat Genet 2019, 51:548-559

44. Barquist L, Mayho M, Cummins C, Cain AK, Boinett CJ, Page AJ, Langridge GC, Quail MA, Keane JA, Parkhill J: The TraDIS toolkit: sequencing and analysis for dense transposon mutant libraries. Bioinformatics 2016, 32:1109-1111
45. Zhu L, Olsen RJ, Nasser W, Beres SB, Vuopio J, Kristinsson KG, Gottfredsson M, Porter AR, DeLeo FR, Musser JM: A molecular trigger for intercontinental epidemics of group A streptococcus. J Clin Invest 2015, 125:3545-3559

46. Bernard PE, Kachroo P, Eraso JM, Zhu L, Madry JE, Linson SE, Ojeda Saavedra M, Cantu C, Musser JM, Olsen RJ: Polymorphisms in regulator of $\mathrm{Cov}$ contribute to the molecular pathogenesis of serotype M28 group A streptococcus. Am J Pathol 2019, 189: 2002-2018

47. Zhu L, Olsen RJ, Horstmann N, Shelburne SA, Fan J, Hu Y, Musser JM: Intergenic variable-number tandem-repeat polymorphism upstream of rocA alters toxin production and enhances virulence in Streptococcus pyogenes. Infect Immun 2016, 84:2086-2093

48. Zhu L, Olsen RJ, Lee JD, Porter AR, DeLeo FR, Musser JM: Contribution of secreted NADase and streptolysin O to the pathogenesis of epidemic serotype M1 Streptococcus pyogenes infections. Am J Pathol 2017, 187:605-613

49. Do H, Makthal N, VanderWal AR, Rettel M, Savitski MM, Peschek N, Papenfort K, Olsen RJ, Musser JM, Kumaraswami M: Leaderless secreted peptide signaling molecule alters global gene expression and increases virulence of a human bacterial pathogen. Proc Natl Acad Sci U S A 2017, 114:E8498-E8507

50. Severin A, Nickbarg E, Wooters J, Quazi SA, Matsuka YV, Murphy E, Moutsatsos IK, Zagursky RJ, Olmsted SB: Proteomic analysis and identification of Streptococcus pyogenes surfaceassociated proteins. J Bacteriol 2007, 189:1514-1522

51. Gallotta M, Gancitano G, Pietrocola G, Mora M, Pezzicoli A, Tuscano G, Chiarot E, Nardi-Dei V, Taddei AR, Rindi S, Speziale P, Soriani M, Grandi G, Margarit I, Bensi G: SpyAD, a moonlighting protein of group A Streptococcus contributing to bacterial division and host cell adhesion. Infect Immun 2014, 82:2890-2901

52. Bombaci M, Grifantini R, Mora M, Reguzzi V, Petracca R, Meoni E, Balloni S, Zingaretti C, Falugi F, Manetti AG, Margarit I, Musser JM, Cardona F, Orefici G, Grandi G, Bensi G: Protein array profiling of tic patient sera reveals a broad range and enhanced immune response against Group A Streptococcus antigens. PLoS One 2009, 4:e6332

53. Horstmann RD, Sievertsen HJ, Knobloch J, Fischetti VA: Antiphagocytic activity of streptococcal M protein: selective binding of complement control protein factor H. Proc Natl Acad Sci U S A 1988, 85:1657-1661

54. Olsen RJ, Sitkiewicz I, Ayeras AA, Gonulal VE, Cantu C, Beres SB, Green NM, Lei B, Humbird T, Greaver J, Chang E, Ragasa WP, Montgomery CA, Cartwright J Jr, McGeer A, Low DE, Whitney AR, Cagle PT, Blasdel TL, DeLeo FR, Musser JM: Decreased necrotizing fasciitis capacity caused by a single nucleotide mutation that alters a multiple gene virulence axis. Proc Natl Acad Sci U S A 2010, 107: 888-893

55. Chochua S, Metcalf BJ, Li Z, Rivers J, Mathis S, Jackson D, Gertz RE Jr, Srinivasan V, Lynfield R, Van Beneden C, McGee L, Beall B: Population and whole genome sequence based characterization of invasive group A streptococci recovered in the United States during 2015. mBio 2017, 8:e01422-17

56. Carey AJ, Weinberg JB, Dawid SR, Venturini C, Lam AK, Nizet V, Caparon MG, Walker MJ, Watson ME, Ulett GC: Interleukin-17A contributes to the control of Streptococcus pyogenes colonization and inflammation of the female genital tract. Sci Rep 2016, 6:26836

57. Soares EM, Mason KL, Rogers LM, Serezani CH, Faccioli LH, Aronoff DM: Leukotriene B4 enhances innate immune defense against the puerperal sepsis agent Streptococcus pyogenes. J Immunol 2013, 190:1614-1622

58. Cook LCC, Chatterjee N, Li Y, Andrade J, Federle MJ, Eichenbaum Z: Transcriptomic analysis of Streptococcus pyogenes colonizing the vaginal mucosa identifies hupY, an MtsR-Regulated adhesin involved in heme utilization. mBio 2019, 10:e00848-19

59. Ong CY, Berking O, Walker MJ, McEwan AG: New insights into the role of zinc acquisition and zinc tolerance in group A streptococcal infection. Infect Immun 2018, 86:e00048-18 
60. Shelburne SA 3rd, Sumby P, Sitkiewicz I, Granville C, DeLeo FR, Musser JM: Central role of a bacterial two-component gene regulatory system of previously unknown function in pathogen persistence in human saliva. Proc Natl Acad Sci U S A 2005, 102:16037-16042

61. Wilk L, Happonen L, Malmstrom J, Herwald H: Comprehensive mass spectrometric survey of Streptococcus pyogenes subcellular proteomes. J Proteome Res 2018, 17:600-617

62. Bateman A, Rawlings ND: The CHAP domain: a large family of amidases including GSP amidase and peptidoglycan hydrolases. Trends Biochem Sci 2003, 28:234-237

63. Fritzer A, Senn BM, Minh DB, Hanner M, Gelbmann D, Noiges B, Henics T, Schulze K, Guzman CA, Goodacre J, von Gabain A, Nagy E, Meinke AL: Novel conserved group A streptococcal proteins identified by the antigenome technology as vaccine candidates for a non-M protein-based vaccine. Infect Immun 2010, 78:4051-4067

64. Reglinski M, Lynskey NN, Choi YJ, Edwards RJ, Sriskandan S: Development of a multicomponent vaccine for Streptococcus pyogenes based on the antigenic targets of IVIG. J Infect 2016, 72:450-459

65. Bensi G, Mora M, Tuscano G, Biagini M, Chiarot E, Bombaci M, Capo S, Falugi F, Manetti AG, Donato P, Swennen E, Gallotta M, Garibaldi M, Pinto V, Chiappini N, Musser JM, Janulczyk R, Mariani M, Scarselli M, Telford JL, Grifantini R, Norais N, Margarit I, Grandi G: Multi high-throughput approach for highly selective identification of vaccine candidates: the Group A Streptococcus case. Mol Cell Proteomics 2012, 11. M111.015693

66. Davies MR, McIntyre L, Mutreja A, Lacey JA, Lees JA, Towers RJ, et al: Atlas of group A streptococcal vaccine candidates compiled using large-scale comparative genomics [Erratum appeared in Nat Genet 2019, 51:1295]. Nat Genet 2019, 51:1035-1043

67. Sitkiewicz I, Green NM, Guo N, Mereghetti L, Musser JM: Lateral gene transfer of streptococcal ICE element RD2 (region of difference 2) encoding secreted proteins. BMC Microbiol 2011, 11:65

68. Deng L, Spencer BL, Holmes JA, Mu R, Rego S, Weston TA, Hu Y, Sanches GF, Yoon S, Park N, Nagao PE, Jenkinson HF, Thornton JA, Seo KS, Nobbs AH, Doran KS: The group B streptococcal surface antigen I/II protein, BspC, interacts with host vimentin to promote adherence to brain endothelium and inflammation during the pathogenesis of meningitis. PLoS Pathog 2019, 15:e1007848

69. Zhang S, Green NM, Sitkiewicz I, Lefebvre RB, Musser JM: Identification and characterization of an antigen I/II family protein produced by group A streptococcus. Infect Immun 2006, 74: $4200-4213$

70. Jain I, Sarkar P, Danger JL, Medicielo J, Roshika R, Calfee G, Ramalinga A, Burgess C, Sumby P: A mobile genetic element promotes the association between serotype M28 group A streptococcus isolates and cases of puerperal sepsis. J Infect Dis 2019, 220: 882-891

71. Nuland S: The Doctors' Plague: Germs, Childbed Fever, and the Strange Story of Ignác Semmelweis. New York, NY, WW Norton, 2003

72. Semmelweis I: Die Aetiologie, der Begriff und die Prophylaxis des Kindbettfiebers [The etiology, concept, and prophylaxis of childbed fever]. German: Madison, University of Wisconsin Press, 1983. Pest, Germany: C. A. Hartleben, 1861 